\title{
Screen-positive rates and agreement among six family history screening protocols for breast/ovarian cancer in a population-based cohort of 21- to 55-year-old women
}

Glenn E. Palomaki, BS ${ }^{1,2}$, Monica R. McClain, PhD ${ }^{1,2}$, Klaus Steinort, $B A^{1,3}$, Randa Sifri, $M D^{4}$, Leigh LoPresti, $M D^{5}$, and James E. Haddow, $M D^{1,2}$

\begin{abstract}
Purpose: Mutations in the BRCA1 and BRCA2 genes are responsible for approximately $2 \%$ of breast cancers by age 70 years. Professional and governmental groups recommend using family history protocols as an initial step in identifying women and families for mutation testing. We assess screen-positive rates and levels of agreement between these protocols. Methods: We applied six family history screening protocols to a population-based cohort of 321 women, age 21 to 55 years, who reported their personal and family history of breast and ovarian cancer. Results: The proportion of women and families identified as candidates for mutation testing ranged from $4.4 \%$ to $7.8 \%$, depending on the protocol. The protocols had low or fair agreement (kappa $<0.75$ for 14 of 15 comparisons), but all identified six women $(1.9 \%, 95 \%$ confidence interval $0.7 \%-4.0 \%)$ as screen positive. When the effect of missing ages of cancer onset was modeled, these rates increased (range 6.5\%-11.5\%), and nine women (2.8\%) were screen positive by all protocols. Conclusion: Given limitations of family history as a screening test for hereditary cancer related to BRCA1/2 mutations, $1 \%$ to $2 \%$ of women in the general population should initially be identified for mutation testing. One way to achieve this would be to require that multiple screening protocols agree. Genet Med 2006:8(3):161-
\end{abstract} 168.

Key Words: breast cancer, family history, screening, general population, BRCA

The most common mutations identified among women with inherited forms of breast/ovarian cancers are in the BRCA1 and $B R C A 2$ genes. Mutations in these genes account for between $1 \%$ and $2 \%$ of all breast cancers by age 70 years. ${ }^{1,2} B R C A 1 / 2$ mutation testing can have a substantial impact on the person being tested, as well as other family members, including such adverse effects as psychologic distress and the fear of social stigmatization..$^{3-12}$ These issues, along with the expense of genetic testing and other considerations, have led to the use of questions about family history for initial evaluation. To date, this approach has been limited to highrisk families and has not been evaluated for routine use in the general population. Recently, the United States Preventive Services Task Force stated that population studies are needed to evaluate the effectiveness of risk stratification when delivered by providers in settings other than referral centers. ${ }^{13}$

\footnotetext{
From the ${ }^{1}$ Foundation for Blood Research, Scarborough, Me; ${ }^{2}$ Women and Infants Hospital, Providence, RI; ${ }^{3}$ Electric Dreams, Inc., Scarborough, Me; ${ }^{4}$ Thomas Jefferson University, Philadelphia, Pa; ${ }^{5}$ Medical College of Wisconsin, Milwaukee, Wis.

Glenn E. Palomaki, Women and Infants Hospital, Department of Pathology and Laboratory Medicine, 70 Elm Street, Providence, RI 02902.

Submitted for publication July 18, 2005.

Accepted for publication December 16, 2005.
}

DOI: 10.1097/01.gim.0000204458.84988.f2
Published family history protocols for identifying inherited forms of breast/ovarian cancer take into account factors such as personal history of cancer, number of affected first- and second-degree relatives, the ages at diagnosis, and ethnicity. Such protocols are intended to identify a small group of women whose evidence of hereditary cancer is sufficiently strong that genetic testing for $B R C A 1 / 2$ mutations can be considered (e.g., positive predictive value of $\geq 10 \%$ ).

The purpose of the present analysis is to (1) identify published breast/ovarian cancer family history screening protocols that are endorsed by professional and/or governmental organizations, (2) apply these protocols to the family histories obtained from a populationbased cohort of younger women, (3) compute and compare the proportion of women identified as candidates for genetic testing by each of the protocols, and (4) compare the level of agreement between the protocols. At least one study has attempted to unify various family history screening protocols, ${ }^{14}$ but, until now, there has been no formal assessment of these protocols using family history information collected at the general population level.

\section{MATERIALS AND METHODS}

\section{Identification of breast/ovarian cancer family history screening protocols}

Family history screening protocols for breast/ovarian cancer were identified primarily through contact with genetics profes- 
sionals and secondarily by a Medline search using the terms "breast cancer," "genetics," and "guidelines." Criteria for inclusion were as follows: (1) the protocol is approved by a professional organization and/or a governmental agency, (2) the protocol is recommended for use in the general population, and (3) the protocol is not directly derived from an existing protocol. We did not include models that were designed primarily to assess the risk of carrying a BRCA1/2 mutation, such as BRCAPRO, ${ }^{15}$ Couch et al.'s, ${ }^{16}$ Shattuck-Eidens et al.'s, ${ }^{17}$ Frank et al.'s, ${ }^{18}$ Manchester scoring system, ${ }^{19}$ or BOADICEA, ${ }^{20}$ because they do not satisfy the first inclusion criterion. All of these models, with the possible exception of the Manchester scoring system, ${ }^{19}$ take considerably more time, effort, and expertise to complete than the family history-taking required by the protocols included in our analyses. Some of these riskprediction models have been reviewed and their applications have been discussed. ${ }^{21,22}$ We also did not include the Family History Assessment Tool ${ }^{23}$ because it does not satisfy the first criterion for inclusion.

\section{Family history data collection}

One of the authors (R.S.) collected personal and family histories of breast/ovarian cancer that included first- and seconddegree relatives from a population-based cohort of women aged 21 to 55 years. The study protocol was approved by the Thomas Jefferson University Institutional Review Board. The questionnaire was designed to be self-administered and was given to women attending an outpatient family medicine clinic. The women did not provide personal identifiers; therefore, it was not possible to use existing patient records to collect any missing information once the questionnaire had been submitted.

\section{Interpretation of a woman's family history}

All protocols were coded into a Visual Basic Program (Microsoft, Redmond, Wash) for analysis. Each protocol was implemented as an independent algorithm. The woman completing the questionnaire was considered to be the index case. In 30 of the families (including all 12 women with a personal history), one age of onset of cancer was unavailable. In 14 additional families, two or more ages of onset were missing. For the initial analysis, age of onset was assumed to be later than all age-associated cutoff levels for the six protocols. Subsequently, a sensitivity analysis was performed by setting the missing values to a random age of onset that was generated separately for breast cancer (Gaussian distribution with a median of 51 years and a standard deviation of 10 years) or ovarian cancer (median 53 years, standard deviation 10 years). These median values were set, conservatively, to 10 years earlier than the median age of onset reported in the latest data reported by the Surveillance, Epidemiology, and End Results. ${ }^{24}$ In 13 families, one or more ages of onset were specified as a decade. In such instances, the age was arbitrarily assigned the midpoint of the range (e.g., "in the 40s" was assigned a value of 44 years). This assumption was also subjected to sensitivity analysis. In two families, the line of descent for a half-sister was not recorded.
They were assumed to be on the side of the family with the more severe history. Again, this assumption was subjected to sensitivity analysis. A half-sibling's history was represented as a second-degree relative. Ambiguities in interpretation were resolved by consensus of a group consisting of a board-certified clinical geneticist, a genetic counselor, and an epidemiologist (M.R.M.).

\section{Limitations of the dataset}

No information was collected regarding Ashkenazi Jewish ethnicity. We assumed all study participants and family members to be non-Jewish. On the basis of the general characteristics of the Jefferson Family Medicine Associates, no more than $3 \%$ of the participating women would be expected to be of Ashkenazi Jewish heritage. No information regarding other forms of cancer (e.g., prostate, colorectal cancer) was collected, because these were rarely considered directly by family history screening protocols.

\section{Statistical analysis}

Each woman's personal and family history was tabulated as being either positive (satisfied at least one criterion in a given protocol) or negative (did not satisfy any criteria in a given protocol). Consistency between protocols was estimated using a nonweighted kappa coefficient by SAS V8.1 (SAS Institute, Inc., Cary, NC). The proportion of participants with a positive result was computed for each protocol. In addition, the proportion of participants positive for one, two, three, four, five, and all six protocols was also computed. Sensitivity analyses were performed for missing ages of onset and missing lines of descent. Confidence intervals (CIs) for proportions were computed using the binomial distribution.

\section{RESULTS}

Six breast/ovarian cancer family history screening protocols were identified that satisfied the inclusion criteria: Wales Cancer Genetic Service (WCGS), ${ }^{25}$ New York State/American College of Medical Genetics (NYS/ACMG), ${ }^{26}$ National Breast Cancer Centre (NBBC), ${ }^{27}$ National Comprehensive Cancer Network (NCCN, v.1.2006), ${ }^{28}$ National Institute for Clinical Excellence (NICE) Quick Reference Guide (referral to tertiary care), ${ }^{29}$ and the United States Preventive Services Task Force (USPSTF). ${ }^{13}$ Although the USPSTF does not identify their referral criteria as a protocol, they are stated as recommendations for referral to genetic counseling for further evaluation. From accompanying literature, the stated purpose of these six protocols was that they be used as an aid in decision-making about BRCA1/2 mutation testing in families. None of the protocols identified in our survey were empirically derived from actual data; instead, the basis for the protocols is expert opinion. A listing of these family history screening protocols is contained in the Appendix.

Over a 3-week period, 488 women between the ages of 21 and 55 years were identified and approached, and 321 (66\%) participated. Details about the 166 women not participating 
Table 1

Proportion of 321 women identified as having a family history indicative of an inherited form of breast/ovarian cancer by each of six published protocols

\begin{tabular}{lcc}
\hline Family history screening protocol & $\begin{array}{c}\text { Number } \\
\text { positive }\end{array}$ & $\begin{array}{c}\text { Percent positive } \\
(95 \% \mathrm{CI})\end{array}$ \\
\hline $\begin{array}{l}\text { New York State/American College of } \\
\quad \text { Medical Genetics }\end{array}$ & 19 & $5.9(3.6-9.1)$ \\
National Breast Cancer Centre, Australia & 18 & $5.6(3.4-8.7)$ \\
Wales Cancer Genetic Services & 16 & $5.0(2.9-8.0)$ \\
$\begin{array}{l}\text { National Institute for Clinical Excellence } \\
\quad \text { tertiary referral) }\end{array}$ & 14 & $4.4(2.4-7.2)$ \\
$\quad$ National Comprehensive Cancer Network & 25 & $7.8(5.1-11.3)$ \\
United States Preventive Services Task Force & 24 & $7.5(4.9-10.9)$ \\
\hline
\end{tabular}

CI, confidence interval.

are not available. Among participants, 203 women (63\%) reported no personal or family history of breast/ovarian cancer, 106 women (33\%) reported a family history but no personal history, and 12 women (4\%) reported a personal history of breast and/or ovarian cancer.

The proportion of women having at least one first-degree relative with breast cancer $(8.3 \%$; $95 \%$ CI $5.6 \%-12 \%)$ is consistent with the $6.4 \%$ reported by others. ${ }^{30}$ Table 1 shows the number and proportion of the 321 women classified as having a family history indicative of an inherited form of breast/ovarian cancer (positive result) for each of the six protocols. Between $14(4.4 \%)$ and $25(7.8 \%)$ of the participating women have a positive screening result, depending on the protocol applied. The positive rates provided in Table 1 are likely to

\section{Table 2}

Proportion of 321 women with negative family histories according to all six breast/ovarian family history screening protocols, along with the number classified as positive by one, two, three, four, five, or all six protocols

\begin{tabular}{lc}
\hline Number of protocols with a positive result & Number of women (\%) \\
\hline None & $282(87.9)$ \\
One only $^{a}$ & $6(1.9)$ \\
Two only $^{b}$ & $17(5.3)$ \\
Three only $^{c}$ & $3(0.9)$ \\
Four only & $d$ \\
Five only & $4(1.2)$ \\
All six & $3(0.9)$ \\
\hline
\end{tabular}

${ }^{a}$ NCCN positive in three, USPSTF positive in two, and NICE positive in one. ${ }^{b} \mathrm{NYS} / \mathrm{ACMG}+$ USPSTF positive in seven, NCCN + NBBC positive in five, WCGS + NCCN positive in two, WCGS + USPSTF positive in two, and NYS/ACMG + NICE positive in one.

${ }^{c}$ One positive each for NCCN + USPSTF + NICE, NBCC + WCGS + NCCN, and NBCC + NCCN + NICE.

${ }^{d}$ One negative each for WCGS + NCCN, NYS/ACMG + WCGS, NBCC + NICE, and NYS/ACMG + USPSTF.

${ }^{e}$ NICE negative in two, and NBCC negative in one. NYS/ACMG, New York State/American College of Medical Genetics; NBCC, National Breast Cancer Centre, Australia; WCGS, Wales Cancer Genetic Services; NICE, National Institute for Clinical Excellence (tertiary referral); NCCN, National Comprehensive Cancer Network; USPSTF, United States Preventive Services Task Force. underestimate the true positive rate, because of our conservative assumption concerning missing ages of cancer onset and the lack of some additional information (e.g., Ashkenazi Jewish heritage).

Table 2 shows that 282 of the 321 women are screen negative by all six protocols ( $87.9 \%)$. It also shows how many are screen positive according to one, two, three, four, five, or six protocols. Each of the rows is mutually exclusive; therefore, 39 of the 321 women (12.1\%) are screen positive on at least one protocol. For example, the fourth row in Table 2 shows that three women are classified as positive by three protocols, but negative by the other three. One of the women in this row had no personal history of cancer, but had a maternal grandmother diagnosed with ovarian cancer at age 83 years and a maternal aunt diagnosed with ovarian cancer at age 61 years. This is a positive family history by the NCCN (maternal aunt satisfies criterion 3a), USPSTF (criterion 1e), and NICE (criterion 2aii) protocols. It is negative according to NYS/ACMG, NBCC, and WCGS protocols. Six women are classified as positive by all six protocols (1.9\%, $95 \%$ CI $0.7 \%-4.0 \%)$. Two had four relatives with breast cancer on the maternal side of the family, and one reported three ovarian cancer cases on the paternal side of the family. Another woman had a mother with early-onset ovarian cancer and a maternal grandmother with early-onset breast cancer. The fifth woman had breast cancer, along with her sister and maternal aunt. The last woman had a sister with breast and ovarian cancer, a son with breast cancer, and a maternal grandmother with early-onset ovarian cancer.

The levels of agreement between all pair-wise combinations of the six protocols are shown in Table 3, using the kappa statistic. Kappa coefficients of less than 0.40 indicate low agreement, coefficients between 0.40 to 0.75 indicate fair agreement, and coefficients greater than 0.75 indicate good agreement. ${ }^{31}$ The analyses were carried out in all 118 women with either a personal history of breast/ovarian cancer or at least one affected family member. One of the lowest levels of agreement is between the NYS/ACMG and NCCN protocols, both of which have relatively high positive rates

Table 3

Level of agreement (kappa statistic) between each pair of the six family history screening protocols estimated using the 118 women with any personal or family history of breast/ovarian cancer

\begin{tabular}{lccccc}
\hline & NBCC & WCGS & NICE & NCCN & USPSTF \\
\hline NYS/ACMG & Low (0.39) & Fair (0.50) & Fair (0.47) & Low (0.33) & Good (0.80) \\
NBCC & - & Fair (0.52) & Low (0.57) & Fair (0.75) & Low (0.37) \\
WCGS & - & - & Low (0.47) & Fair (0.62) & Fair (0.52) \\
NICE & - & - & - & Fair (0.49) & Fair (0.44) \\
NCCN & - & - & - & - & Low $(0.36)$ \\
\hline
\end{tabular}

NYS/ACMG, New York State/American College of Medical Genetics; NBCC National Breast Cancer Centre, Australia; WCGS, Wales Cancer Genetic Services; NICE, National Institute for Clinical Excellence (tertiary referral); NCCN, National Comprehensive Cancer Network; USPSTF, United States Preventive Services Task Force. 
(from Table 1, 19 and 25 women positive for the NYS/ ACMG and NCCN protocols, respectively). However, only 10 women are identified as positive by both protocols. The highest level of agreement is between the NYS/ACMG and USPSTF protocols. These protocols classify 19 and 24 women, respectively, as being screen positive. Eighteen of the 19 women identified as positive by the NYS/ACMG protocol are also positive by the USPSTF protocol. It is not known whether the level of agreement between the protocols is related to their ability to predict BRCA1/2 mutation risk.

Table 4 shows how selected risk factors associated with inherited forms of breast/ovarian cancer are used by the six family history screening protocols. Three of the factors are used by all six protocols (total number of breast cancer cases, bilateral breast cancer, and a male with breast cancer). However, the protocols differ in how these factors are used. For example, bilateral breast cancer alone in any family member is considered sufficient to classify a woman as screen positive by the NYS/ACMG protocol, whereas WCGS and USPSTF protocols require that the bilateral breast cancer be in a first-degree relative; the NICE protocol requires the cancers to occur at an average age earlier than 50 years, whereas NBCC and NCCN protocols require an additional family member with cancer on the same side of the family. There is less agreement about whether Ashkenazi Jewish heritage should be considered (NICE and WCGS do not address this criterion). Other types of cancers are directly addressed only by the NBCC and NICE protocols.

In this dataset, ages of cancer onset are missing in 44 family histories, and the analyses shown so far assume them all to be at 55 years of age (older than the highest cutoff level used in any of the six protocols). To estimate the maximum impact of this age factor on the positive rate, we then randomly assigned ages of onset for those that were missing (as described earlier). These ages are, on average, younger than expected in the general population by 10 years. This was done to account for the fact that, in all instances, there is at least some family history already present, and to set a reasonable "upper limit" to the screen-positive rates. As expected, the number of screen-positive women increases for all six protocols. Two protocols sustained only modest increases (USPSTF from 24 to 25 and NYS/ACMG from 19 to 22 ). The four other protocols increased more dramatically (WCGS from 15 to 21, NBCC from 18 to 25, NICE from 15 to 22 , and NCCN from 25 to 37 ). The lower number of positives (Table 2) likely represents screening expectation in primary care, where missing data are expected. The higher number of positives found by modeling might be approached if time and resources are made available to more carefully document family history in primary care settings.

In 20 additional instances, ages of cancer onset were provided only as a decade, and the analyses shown so far assume each to be at the middle of the designated decade. To estimate the maximum and minimum impact of this assumption, the ages are first set to the upper limit of the specified range and then to the lower limit. The only change occurs for the NCCN protocol, in which the number of women classified as positive increases from 25 to 26 when the ages are set to the lower limit. In two families, the line of descent was not provided for a half-sister, and the previous analyses assume her to be on the side of the family with the strongest family history. To examine the impact of this assumption, all are now set to the other side of the family. This yields a reduction in the number of women classified as positive by two of the protocols: NBCC (from 18 to 17) and NCCN (from 25 to 23). These types of missing information, therefore, would have little impact on the proportion of women with positive family histories.

Table 4

How selected risk factors associated with inherited forms of breast/ovarian cancer are used in six family history screening protocols

\begin{tabular}{|c|c|c|c|c|c|c|c|}
\hline \multirow[b]{2}{*}{ Factor(s) needed for "positive" } & \multicolumn{6}{|c|}{ Family history screening protocol } & \multirow[b]{2}{*}{ Protocols using factor } \\
\hline & NYS/ACMG & NBCC & WCGS & NICE & $\mathrm{NCCN}$ & USPSTF & \\
\hline Number of BCs at any age $^{a}$ & $>3$ & $>3$ & $>3$ & $>3$ & $>3$ & $>3^{a}$ & 6 \\
\hline Bilateral $\mathrm{BC}$ alone & Yes & If a second case & If $1^{\circ}$ & If $<50 y$ & If a second case & If $1^{\circ}$ & 6 \\
\hline Male with BC & Yes & If a second case & Yes & If a second case & If a second case & Yes & 6 \\
\hline Age defining "early-onset" BC & $<45$ & $<40$ & $<40$ & $<40$ & $<40$ & No & 5 \\
\hline $\mathrm{BC}$ and $\mathrm{OC}$ in one relative & No & If a second case & If $1^{\circ}$ & No & Yes & Yes & 4 \\
\hline Consider Ashkenazi Jewish heritage & Yes & Yes & No & No & Yes & Yes & 4 \\
\hline Consider identified family mutations & Yes & Yes & No & No & Yes & $\mathrm{No}^{b}$ & 3 \\
\hline Consider personal history of $\mathrm{BC} / \mathrm{OC}$ & Yes & No & No & No & Yes & No & 2 \\
\hline Consider other cancers & No & Yes & No & Yes & $\mathrm{No}^{c}$ & No & 2 \\
\hline
\end{tabular}

${ }^{a}$ All but the USPSTF protocol require the affected individuals to be on the same side of the family.

${ }^{b}$ Text accompanying the protocol indicates that women with a known BRCA mutation may be referred for genetic counseling.

${ }^{c}$ Footnote mentions that the presence of other cancers (prostate, pancreatic, or melanoma) may increase suspicion BC, breast cancer; OC, ovarian cancer; NYS/ ACMG, New York State/American College of Medical Genetics; NBCC, National Breast Cancer Centre, Australia; WCGS, Wales Cancer Genetic Services; NICE, National Institute for Clinical Excellence (tertiary referral); NCCN, National Comprehensive Cancer Network; USPSTF, United States Preventive Services Task Force. 


\section{DISCUSSION}

Others have commented on the lack of consistent criteria among family history protocols, ${ }^{14}$ but this is the first time that the extent of agreement has been documented using real patient data. Although one of the comparisons (NYS/ACMG and USPSTF) showed good agreement, the other 14 comparisons showed only low to fair agreement among the six protocols. Several other important performance features also emerged: (1) ensuring that all ages at onset of cancer are available is important for accurately interpreting family histories for most of the protocols, (2) the proportion of women classified as having a positive family history varies substantially among protocols, (3) each of the protocols individually classifies a relatively high proportion of women as having a positive family history, and (4) all six protocols agree that a small group of women has a positive family history.

All six of the protocols evaluated in this study are designed to be applied in the general population. At present, however, no formal pilot trials have been carried out to assess their performance. A positive screening test carries with it serious implications for the woman and her family from an emotional, financial, and medical point of view, and any negative impact will be magnified when screening is extended to the general population. Under such circumstances, it would be prudent to design intervention trials to identify only the families with the strongest evidence of an inherited form of cancer as screen positive until the screening test's performance characteristics can be better understood. Some guidelines and at least one professional organization have recommended that family history screening protocols be sufficiently discriminatory to ensure that at least 1 in 10 women (10\%) classified as having a positive family history will carry a BRCA1/2 mutation. ${ }^{32}$ This $10 \%$ positive predictive value seems to be reasonable as a starting point, and the present study provides data to help determine how this might be accomplished.

In the general population, approximately 10 in every 3,500 women carry one of these mutations (excluding Ashkenazi Jewish women, among whom the carrier rate is higher because of founder mutations). ${ }^{1}$ By assuming family history to be positive in all 10 of the women, the positive predictive value of $10 \%$ would be reached by classifying 100 of the screened women as candidates for mutation testing ( $[10$ true positives +90 false positives $] / 3,500=2.9 \%)$. However, this $100 \%$ detection rate is likely to be an overestimate because of inherent limitations of using family history as a screening test. These limitations are apparent in a review of four published studies that collectively carried out mutation testing on 1,667 younger women with breast cancer. Among these women, 431 (26\%) also had at least one affected first-degree relative. ${ }^{1,33-35}$ Even among those with a BRCA1/2 mutation, only 6 of 10 had a first-degree relative with breast/ovarian cancer. In our study, only $4 \%$ of women had breast cancer, suggesting that fewer than six might be detected. However, we collected a more extensive family history (both first- and second-degree relatives), which might result in higher detection. Taken together, the evidence leads us to conclude that between 4 and 6 of every 10 women in the general population with a BRCA1/2 mutation might have a positive family history. If family history could detect 4 of the 10 women with mutations in a primary care setting, the acceptable number of screen positives would be 40 per 3,500 women (1.1\%). If family history could detect 6 of the 10 mutations, the total number of screen positives could be increased to $60(1.7 \%)$.

An alternative approach would be to reduce the threshold defining a positive family history as a way to increase the identification of families carrying deleterious mutations (e.g., $12.1 \%$ of the women in our study were screen positive by at least one protocol) (Table 2). The small gain in women identified with a mutation, however, would be offset by a disproportionately large number of women who would require genetic counseling and, should they proceed with genetic testing, receive negative (or uninformative) $B R C A 1 / 2$ mutation test results. Also, these women would not be candidates for primary preventive action, unlike the small number of women with an extensive family history. The additional medical, financial, and emotional impact of such an approach should be examined carefully before any such introduction.

Several characteristics of these screening protocols deserve further discussion. The USPSTF protocol explicitly excludes women with a personal history of breast and/or ovarian cancer. To make a more fair comparison among protocols, we considered the 12 women with a personal history to be first-degree relatives instead of removing them from the screening process entirely. In practice, excluding these women (and their families) would reduce the usefulness and overall performance of family history screening. The NYS/ACMG and USPSTF protocols tend to agree more (and tend to have higher positive rates) mainly because of criteria that include one case of breast cancer (at any age) and another case of ovarian cancer (at any age) to define a screen positive. In general, we found that family histories that are positive according to one, two, three, or four protocols are usually positive for only a single criterion on each. In contrast, the family histories positive according to five or six protocols are often positive for multiple criteria in each protocol. The NICE organization has promulgated two separate family history screening protocols, one relatively inclusive protocol to be used for referrals from primary care to secondary care and another to be used for referrals from secondary to tertiary care. We chose to implement the latter, because women positive at the tertiary level are likely to be offered mutation testing. We encountered one difficulty during implementation. The protocol contained in the quick reference guide does not match the protocol provided in the full clinical guidelines. We chose to implement the former, because it seems to be designed for use in practice. The NICE protocol uses the average age of onset of cancer. This cannot be computed if even one age is missing. The other protocols do not call for average age, and missing data are less problematic for them. Our modeling of missing ages of onset most strongly affects the NCCN protocol, because a single family member diagnosed with breast cancer at or before age 40 years is sufficient to 
define a positive family history. Other protocols require additional factors to be present.

If family history is to be systematically introduced as a screening test for hereditary breast/ovarian cancer, a prime consideration should be to establish a highly selective policy for classifying women as candidates for BRCA1/2 mutation testing. This is especially true in the early stages of screening program development. One possible way to achieve that goal might be to require that several family history screening protocols agree. The present study, for example, indicates that a $1.9 \%$ screen-positive rate might be achieved when six family history screening protocols all agree. This is consistent with the estimated acceptable range for screen positive rates of $1 \%$ to $2 \%$. Currently, it is not possible to determine the actual screening performance of any of these protocols. Instead, we view each of them as an "expert opinion" and the combination of the protocols as an "expert panel." It is unrealistic to expect that BRCA1/2 mutation test results will be available from an entire population-based cohort of women who have family history available, even though such a study would allow the detection and false-positive rates to be computed and the actual performance of the protocols to be directly compared. As an intermediate step in helping to define performance, it would be more realistic to apply a set of family history screening protocols to a cohort of women from the general population and determine the proportion of screen-positive women/ families with a detectable mutation (positive predictive value).

\section{ACKNOWLEDGMENTS}

Support for collecting background information for this study was provided by a cooperative agreement (UR3/CCU319352) with the Centers for Disease Control and Prevention, and Office of Genomics and Disease Prevention.

\section{References}

1. Peto J, Collins N, Barfoot R, SealS, et al. Prevalence of BRCA1 and BRCA2 gene mutations in patients with early-onset breast cancer. J Natl Cancer Inst 1999;91:943949.

2. McClain MR, Palomaki GE, Nathanson KL, Haddow JE. Adjusting the estimated proportion of breast cancer cases associated with BRCA1 and BRCA2 mutations: public health implications. Genet Med 2005;7:28-33.

3. Cho MK, Sankar P, Wolpe PR, Godmilow L. Commercialization of BRCA1/2 testing: practitioner awareness and use of a new genetic test. Am J Med Genet 1999;83: 157-163.

4. Croyle RT, Smith KR, Botkin JR, Baty B, et al. Psychological responses to BRCA1 mutation testing: preliminary findings. Health Psychol 1997;16:63-72.

5. Dudok de Wit AC, Tibben A, Frets PG, Meijers-Heijboer, et al. Males at-risk for the BRCA1-gene, the psychological impact. Psychooncology 1996;5:251-257.

6. Hallowell N, Foster C, Ardern-Jones A, Eeles R, et al. Genetic testing for women previously diagnosed with breast/ovarian cancer: examining the impact of BRCA1 and BRCA2 mutation searching. Genet Test 2002;6:79-87.

7. Lehmann LS, Weeks JC, Klar N, Garber JE. A population-based study of Ashkenazi Jewish women's attitudes toward genetic discrimination and BRCA1/2 testing. Genet Med 2002;4:346-352.

8. Lerman C, Biesecker B, Benkendorf JL, Kerner J, et al. Controlled trial of pretest education approaches to enhance informed decision-making for BRCA1 gene testing. J Natl Cancer Inst 1997;89:148-157.

9. Lerman C, Hughes C, Lemon SJ, Main D, et al. What you don't know can hurt you: adverse psychologic effects in members of BRCA1-linked and BRCA2-linked families who decline genetic testing. J Clin Oncol 1998;16:1650-1654.

10. Lerman C, Narod S, Schulman K, Hughes C, et al. BRCA1 testing in families with hereditary breast-ovarian cancer. A prospective study of patient decision making and outcomes. JAMA 1996;275:1885-1892.
11. Lynch HT, Lemon SJ, Durham C, Tinley ST, et al. A descriptive study of BRCA1 testing and reactions to disclosure of test results. Cancer 1997;79:2219-2228.

12. Schwartz MD, Peshkin BN, Hughes C, Main D, et al. Impact of BRCA1/BRCA2 mutation testingon psychologic distress in a clinic-based sample. J Clin Oncol 2002; 20:514-520.

13. Genetic risk assessment and BRCA mutation testing for breast and ovarian cancer susceptibility: recommendation statement. Ann Intern Med 2005;143:355-361.

14. Hampel H, Sweet K, Westman JA, Offit K, et al. Referral for cancer genetics consultation: a review and compilation of risk assessment criteria. J Med Genet 2004;41: 81-91.

15. Parmigiani G, Berry D, Aguilar O. Determining carrier probabilities for breast cancer-susceptibility genes BRCA1 and BRCA2. Am J Hum Genet 1998;62:145-158.

16. Couch FJ, DeShano ML, Blackwood MA, Calzone K, et al. BRCAl mutations in women attending clinics that evaluate the risk of breast cancer. N Engl J Med 1997; 336:1409-1415.

17. Shattuck-Eidens D, Oliphant A, McClure M, McBride C, et al. BRCAl sequence analysis in women at high risk for susceptibility mutations. Risk factor analysis and implications for genetic testing. JAMA 1997;278:1242-1250.

18. Frank TS, Manley SA, Olopade OI, Cummings S, et al. Sequence analysis of BRCA1 and BRCA2: correlation of mutations with family history and ovarian cancer risk. J Clin Oncol 1998;16:2417-2425.

19. Evans DG, Eccles DM, Rahman N, Young K, et al. A new scoring system for the chances of identifying a BRCA1/2 mutation outperforms existing models including BRCAPRO. J Med Genet 2004;41:474-480.

20. Antoniou AC, Pharoah PP, Smith P, Easton DF. The BOADICEA model of genetic susceptibility to breast and ovarian cancer. Br J Cancer 2004;91:1580-1590.

21. Domchek SM, Eisen A, Calzone K, Stopfer J, et al. Application of breast cancer risk prediction models in clinical practice. J Clin Oncol 2003;21:593-601.

22. Nelson HD, Huffman LH, Fu R, Harris EL. Genetic risk assessment and BRCA mutation testing for breast and ovarian cancer susceptibility: systematic evidence review for the U.S. Preventive Services Task Force. Ann Intern Med 2005;143:362-379.

23. Gilpin CA, Carson N, Hunter AG. A preliminary validation of a family history assessment form to select women at risk for breast or ovarian cancer for referral to a genetics center. Clin Genet 2000;58:299-308.

24. Ries L, Eisner M, Kosary C, Hankey B, et al. SEER Cancer Statistics Review, 1975 2002. National Cancer Institute, Bethesda, MD. Available at: http://seer.cancer.gov/ csr/1975-2002/, based on November 2004 SEER data submission, posted to the SEER web site 2005. Accessed 1 December 2005.

25. Elwyn G, Iredale R, Gray J. Reactions of GPs to a triage-controlled referral system for cancer genetics. Fam Pract 2002;19:65-71.

26. Genetic susceptibility to breast and ovarian cancer: assessment, counseling and testing guidelines: American College of Medical Genetics Foundation, 1999. Available at: http://www.health.state.ny.us/nysdoh/cancer/obcancer/contents.htm. Accessed 1 December 2005.

27. Advice about familial aspects of breast cancer and ovarian cancer: a guide for health professionals: National Health and Medical Research Council, National Breast Cancer Centre, 2000. Available at: http://www.nbcc.org.au/bestpractice/resources/ BOG_BreastOvarianGuideSimpl.pdf. Accessed 1 December 2005.

28. Genetic/familial high-risk assessment: breast and ovarian. Clinical practice guidelines in oncology - v. 1.2004. Jenkintown, PA: National Comprehensive Cancer Network, 2004. Genetic/familial high-risk assessment: breast and ovarian Version 1.2006. Jenkintown, PA: National Comprehensive Cancer Network. Available at: http://www.nccn.org/professionals/physician_gls/. Accessed 1 December 2005.

29. Clinical guidelines for the classification and care of women at risk of familial breast cancer: National Institute for Clinical Excellence, 2003: 40-41. Clinical guidelines for the classification and care of women at risk of familial breast cancer: National Institute for Clinical Excellence, UK; June, 2004. Available at: http://www.nice.org.uk/ page.aspx?o=guidelines.completed. Accessed 1 December 2005.

30. Hall IJ, Burke W, Coughlin S, Lee NC. Population-based estimates of the prevalence of family history of cancer among women. Community Genet 2001;4:134-142.

31. Fleis JL. Statistical Methods for Rates and Proportions. New York: John Wiley \& Sons, 1981.

32. Statement of the American Society of Clinical Oncology: genetic testing for cancer susceptibility. Adopted on February 20, 1996. J Clin Oncol 1996;14:1730-1736; discussion 1737-1740.

33. Malone KE, Daling JR, Neal C, Suter NM, et al. Frequency of BRCA1/BRCA2 mutations in a population-based sample of young breast carcinoma cases. Cancer 2000; 88:1393-1402.

34. Loman N, Johannsson O, Kristoffersson U, Olsson H, et al. Family history of breast and ovarian cancers and BRCA1 and BRCA 2 mutations in a population-based series of early-onset breast cancer. J Natl Cancer Inst 2001;93:1215-1223.

35. Hopper JL, Southey MC, Dite GS, Jolley DJ, et al. Population-based estimate of the average age-specific cumulative risk of breast cancer for a defined set of protein- 
truncating mutations in BRCA1 and BRCA2. Australian Breast Cancer Family Study. Cancer Epidemiol Biomarkers Prev 1999;8:741-747.

\section{Appendix}

Definitions of a positive family history of breast/ovarian cancer according to six published screening protocols (These protocols have been reconfigured and presented in a consistent format to make reading and comparisons easier.)

\section{A. New York State Department of Health and American College of Medical Genetics (NYS/ACMG) Genetic Susceptibility to Breast and Ovarian Cancer Assessment, Counseling and Testing Guidelines (1999)}

1. Three or more affected $1^{\circ}$ or $2^{\circ}$ relatives with breast or ovarian on the same side of the family, regardless of age at diagnosis OR

2. Fewer than three affected relatives but one or more of the following:

a) The patient was diagnosed at $\leq 45$ years

b) A family member has been identified with a detectable mutation

c) There are one or more cases of ovarian cancer at any age, and one or more members on the same side of the family with breast cancer at any age

d) There are multiple primary or bilateral breast cancers in the patient or one family member

e) There is breast cancer in a male patient, or in a male relative

f) A patient is at increased risk for specific mutations(s) because of ethnic background (i.e., Ashkenazi Jewish descent) and has one or more affected relatives with breast or ovarian cancer at any age

B. Management of Early Breast Cancer, National Breast Cancer Centre, Australia (NBCC) Clinical Practice Guidelines (2000)

1. Two $1^{\circ}$ or $2^{\circ}$ relatives on one side of the family diagnosed with breast or ovarian cancer plus one or more of the following features on the same side of the family:

a) Additional relative(s) with breast or ovarian cancer

b) Breast cancer diagnosis at $<40$ years

c) Ovarian cancer diagnosed $<50$ years

d) Bilateral breast cancer

e) Breast and ovarian cancer in the same individual

f) Jewish ancestry

g) Breast cancer in a male

2. One $1^{\circ}$ or $2^{\circ}$ relative diagnosed with breast cancer at $\leq 45$ years plus another $1^{\circ}$ or $2^{\circ}$ relative on the same side of the family with sarcoma (bone/soft tissue) at $\leq 45$ years

3. Member of a family in whom the presence of a high-risk breast cancer gene mutation has been established

\section{All Wales Cancer Genetics Service (WCGS) Referral Guidelines (2002)}

1. Breast cancer (and one or more of the following)

a) One $1^{\circ}$ relative diagnosed $\leq 40$ years b) Two $1^{\circ}$ relatives diagnosed $\leq 60$ years (on the same side of the family)

c) Three $1^{\circ}$ or $2^{\circ}$ relatives diagnosed any age (on the same side of the family)

d) One $1^{\circ}$ male with breast cancer diagnosed at any age

e) One $1^{\circ}$ relative with bilateral breast cancer

2. Breast/ovarian cancer (and one or more of the following)

a) One or more occurrences of ovarian cancer and one or more occurrences of breast cancer on the same side of the family (if only one of each, the breast cancer diagnosed $<50$ years $)$

b) $\mathrm{A} 1^{\circ}$ relative with both breast and ovarian cancer

3. Ovarian cancer

a) Two or more relatives (on the same side of the family) and one is a $1^{\circ}$ relative

D. National Institute for Clinical Excellence (NICE) Familial Breast Cancer Clinical Guideline 14 (2004): Quick Reference Guide

Referral criteria from secondary care to tertiary care

1. Female breast cancers only (on the same side of the family)

a) One $1^{\circ}$ and one $1^{\circ}$ or $2^{\circ}$ relative with breast cancer diagnosed at average age $<50$ years

b) One $1^{\circ}$ and two $1^{\circ}$ or $2^{\circ}$ relatives with breast cancer diagnosed at average age $<60$ years

c) One $1^{\circ}$ and three (or more) $1^{\circ}$ or $2^{\circ}$ relatives with breast cancer diagnosed at any age

2. Ovarian cancer

a) One relative with ovarian cancer at any age and on the same side of the family

i) One $1^{\circ}$ (including relative with ovarian cancer) or one $2^{\circ}$ relative with breast cancer diagnosed $<50$ years

ii) One additional relative diagnosed with ovarian cancer at any age

iii) Two $1^{\circ}$ or $2^{\circ}$ relatives with breast cancer diagnosed $<60$ years

3. Bilateral breast cancer (on the same side of the family)

a) One $1^{\circ}$ relative with cancer diagnosed in both breasts at an average age $<50$ years

b) One $1^{\circ}$ or $2^{\circ}$ relative with bilateral breast cancer and one $1^{\circ}$ or $2^{\circ}$ relative diagnosed with breast cancer before average age of 60 years

4. Male breast cancer (on the same side of the family)

a) One male breast cancer at any age and

i) One $1^{\circ}$ or $2^{\circ}$ relative with breast cancer diagnosed $<50$ years

ii) Two $1^{\circ}$ or $2^{\circ}$ relatives with breast cancer diagnosed at an average age $<60$ years

\section{E. National Comprehensive Cancer Network, v.1.2006 (NCCN) Practice Guidelines (2005)}

1. Member of a family with a known BRCA1/BRCA2 mutation

2. Personal history of breast cancer plus one or more of the following:

a) Diagnosed $\leq 40$ years, with or without family history

b) Diagnosed $\leq 50$ years, or bilateral, with at least one close $^{\star}$ blood relative with breast cancer diagnosed $\leq 50$ 
years or at least one close blood relative with ovarian cancer

c) Diagnosed at any age, with at least two close ${ }^{\star}$ blood relatives with ovarian cancer at any age

d) Diagnosed at any age with breast cancer with at least two close ${ }^{\star}$ blood relatives with breast cancer, especially if at least one is diagnosed before age 50 years or has bilateral disease

e) Close* male blood relative has breast cancer

f) Personal history of ovarian cancer

g) Is of ethnic descent associated with deleterious mutations (e.g., Ashkenazi Jewish)

3. Personal history of ovarian cancer plus one or more of the following:
a) At least one close ${ }^{\star}$ blood relative with ovarian cancer
b) At least one close ${ }^{\star}$ female blood relative with breast can- cer at age $\leq 50$ years or bilateral breast cancer
c) At least two close ${ }^{\star}$ blood relatives with breast cancer
d) At least one close ${ }^{\star}$ male blood relative with breast cancer
e) Is of Ashkenazi Jewish descent

4. Personal history of male breast cancer plus one or more of the following:
a) At least one close ${ }^{\star}$ male blood relative with breast cancer
b) At least one close ${ }^{\star}$ female blood relative with breast or ovarian cancer

c) Ashkenazi Jewish descent

5. Family history only: close ${ }^{\star}$ family member (on the same side of the family) meeting any of the above criteria

${ }^{*}$ Close is defined by the NCCN protocol as first-, second-, and third-degree relatives.

\section{F. United States Preventive Services Task Force Clinical Guidelines for Women with No Personal History of Breast/Ovarian Cancer USPSTF (2005)}

1. Among non-Ashkenazi Jewish women

a) Two $1^{\circ}$ relatives with breast cancer; one of whom was diagnosed $\leq 50$ years

b) Three or more $1^{\circ}$ or $2^{\circ}$ relatives with breast cancer

c) Both breast and ovarian cancer among $1^{\circ}$ and $2^{\circ}$ relatives

d) A first-degree relative with bilateral breast cancer

e) Two or more $1^{\circ}$ or $2^{\circ}$ relatives with ovarian cancer

f) $\mathrm{A} 1^{\circ}$ or $2^{\circ}$ relative with both breast and ovarian cancer

g) Breast cancer in a male relative

2. Among Ashkenazi Jewish women

a) A $1^{\circ}$ relative with breast or ovarian cancer

b) Two $2^{\circ}$ relatives on the same side of the family with breast or ovarian cancer 3 . Relative with a known deleterious mutation in BRCA1 or BRCA2 